\title{
Dielectrophoresis-based microfluidic device for separation of potential cancer cells
}

\author{
Nur Fatien Najwa Mohamad Narji, Mohd Ridzuan Ahmad \\ Division of Control and Mechatronics Engineering, School of Electrical Engineering, Faculty of Engineering, \\ Universiti Teknologi Malaysia, Johor, Malaysia
}

\begin{abstract}
Article Info
Article history:

Received Feb 13, 2020

Revised Apr 19, 2020

Accepted May 7, 2020

\section{Keywords:}

Cancerous cell

CT scanning

Dielectrophoresis

Mammography

Microfluidic

ABSTRACT

Cancer is a leading cause of death that adversely affects all ages and genders around the world. There is a range of approaches such as CT scanning and mammography to diagnose cancer. Although the current method has many benefits, most of it share similar drawbacks as the result of detection takes long time and can lead to over diagnosis. Dielectrophoresis (DEP) is a method that can be used to obtain the cell electrical properties such as capacitance, conductivity, and permittivity. A device was designed in this study using a pair of electrodes and main channel with two inlets and two outlets. COMSOL software is adopted to analyze channel particle flow. Results show the configuration of microfluidic device and its dimensions. For potential application, DEP may be used as a non-invasive technique to distinguish normal cell from cancerous cell, which can lead to early detection as it offers a real-time warning. The simulations reveal that the electrodes captured the particles successfully and sorted them within specific time. The chance of cell capture and the ability of the electrodes to sort the cells is around $80 \%$. In addition, an ideal design of the microfluidic chip was established, incorporating the cell and dielectric properties.
\end{abstract}

This is an open access article under the CC BY-SA license.

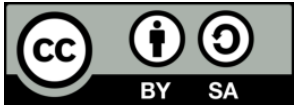

\section{Corresponding Author:}

Mohd Ridzuan Ahmad,

Division of Control and Mechatronic Engineering,

School of Electrical Engineering, Faculty of Engineering,

Universiti Teknologi Malaysia,

81310 Skudai, Johor, Malaysia.

Email: mdridzuan@utm.my

\section{INTRODUCTION}

This paper focuses on a blood cell separation device. The system of this device can detect and separate the cells by using the dielectric properties and produce output on real-time notifications. Cancer is a disease caused by the growth of abnormal cell in our body [1]. Unlike normal cells, cancer cells do not stop growing. The cells keep doubling and forming a tumor that grows in size. Cancer of the blood cells does not form a tumor, but eventually, they create an abnormal cell in our blood. Cancer risk factors can be roughly divided into a biological factor, environmental exposure, occupational risk, and lifestyle-related factor [2]. With a higher condition of being diseased and a higher death rate, cancer has become an essential health threat problem around the world.

According to the National Cancer Institute, the number of new cancer cases is expected to increase from about 1.5 million per year in 2010 to 1.9 million per year in 2020 [3]. While for the number of cancer deaths, it is expected to increase from about 575,000 per year in 2010 to 630,000 per year in 2020. In 2018, it is estimated that $18,078,957$ new cases recorded for all cases combined. The most crucial part is more than half of the new cases cause death. 
This paper focuses to develop a simulation of a microfluidic system that can detect cancerous cell in a blood sample. The main properties to simulate the system are by determining the dielectric properties and the types of the electrode that will be used in the system. Nowadays, there are a lot of conventional methods to detect cancer at an early stage. Some of the popular methods are blood test, clinical imaging and biopsies [4]. A blood test commonly tests and measures the amount of varies types of blood cell in the sample. Next, screening test methods for clinical application include ultrasonic testing, X-ray imaging, computed tomography (CT) [2], magnetic resonance imaging (MRI) [5], mammography [6] and Endoscope [7]. On the other hand, a biopsy is a sample of abnormal tissue taken from the body. Although all of the conventional methods have their own advantages, there are also some disadvantages to these approaches [8].

For example, the blood test cannot give the final result and cannot give confirmation whether the patients have a disease or not. A blood test does not provide detailed information about cancer detection [9]. They only provide some clues by identifying the cancer markers (biomarkers). This is shows that the blood test can only provide qualitative data that leads to insufficient data to detect cancerous cells. Next, for clinical imaging, usually, the results are lagging behind the tumor progression. Research shows that specific screening test such as Mammography takes around three days to weeks depending on the critical stage of cancer [10], while for the analysis of biopsies, it takes more than 10 days to give the result [11]. Then, the clinical imaging method uses high technologies such as radiation and high magnetization force, so this leads to bigger investment and higher cost for the patient to pay [12]. As for the biopsy technique, it can reach more than RM5, 000 per session depending on types of biopsies. Biopsies also can cause altered appearances depends on the size of tissue taken [13].

The most important measures of diagnostic accuracy are sensitivity and specificity. Sensitivity refers to the ability of the test to identify patients with the disease. Specificity refers to the ability of the test to identify patients without the disease [14]. According to an article written by Y Toyoda [15], sensitivity and specificity by the screening detection of lung cancer were 88.9 and $92.6 \%$ for low-dose CT and 78.3 and $97.0 \%$ for chest X-ray, respectively. The sensitivity of low-dose CT by the incidence method was $79.5 \%$, whereas that of the chest X-ray was $86.5 \%$. Thus, it is necessary to find a new method, which can give higher accuracy of quantitative data instead of qualitative data [10]. It is also fundamental to develop a method that can give real-time notification to detect cancer at an early stage

\section{RESEARCH METHOD}

The device is fabricated as three layers microfluidic chip as shown in Figure 1(a). The device has two inlet ports, which are for buffer solution and blood sample, two outlet ports, housing a resistor ladder network, interconnection chamber electrodes. The device integrates and employs hydrodynamic focusing at the cell injector ports. Outlet ports are connected to two identical syringes housed on a variable speed syringe pump. At the end of the channel, the fluid and separated cell are directed to two identical but separate outlets for sample collection. It is designated in a miniature device with the dimension as illustrated in Figure 1(b). For the second and third layer, the length is $89 \mathrm{~mm}$. For the cover plate extension, the length is $6 \mathrm{~mm}$ longer and for the base plate extension, it is $10 \mathrm{~mm}$ wider. The main channel is around $600 \mathrm{micro} \mathrm{mm}$. The overall dimension is $9 \mathrm{~cm} \times 3 \mathrm{~cm}$.

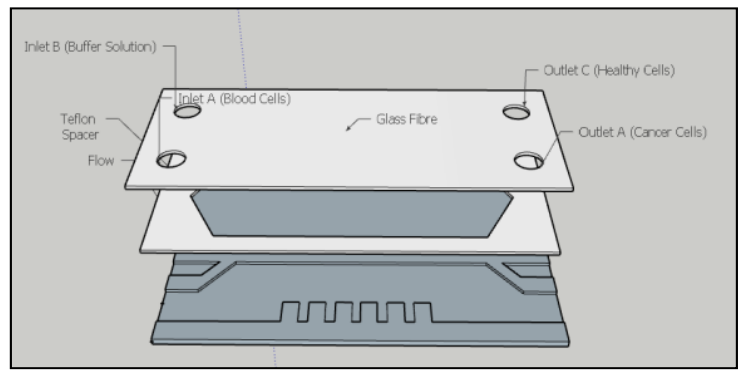

(a)

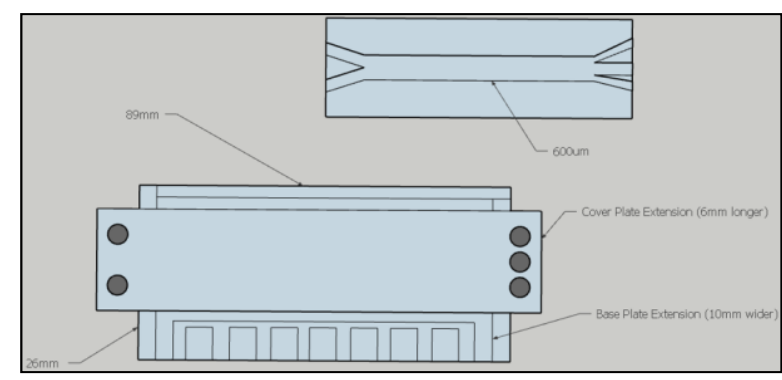

(b)

Figure 1. The proposed design of the microfluidic chip, (a) The overview of the microfluidic chip design, (b) The dimension of microfluidic chip design

In this study, the dielectric properties of red blood cells and cancerous cells were measured. The particles diameter of the cancerous cell is approximately two times smaller than normal blood cells. All the dielectric properties were fixed as shown in Tables 1 and 2. The dielectric properties consist of 
diameter, conductivity, permittivity, and viscosity. The formula used will be explained in the next part of this journal. Dielectrophoretic (DEP) phenomena occur when particles in a medium are exposed to a non-uniform field and cause polarization for particular particles according to their dielectric properties [16]. DEP Force is classified into two types according to correlations of dielectric properties of both particles and medium [17].

Table 1. Parameters of cancerous cells and RBCs

\begin{tabular}{llll}
\hline Variables & Cancerous cell & RBC & Unit \\
\hline Particle diameter & 2.4 & 5 & Um \\
Particle conductivity & 0.25 & 0.31 & $\mathrm{~S} / \mathrm{m}$ \\
Particle relative permittivity & 50 & 59 & \\
Shell electrical conductivity & $1.00 \mathrm{E}-06$ & $1.00 \mathrm{E}-06$ & $\mathrm{~S} / \mathrm{m}$ \\
Shell relative permittivity & 6 & 4.44 & \\
Shell thickness & 8 & 9 & $\mathrm{~nm}$ \\
\hline
\end{tabular}

Table 2. Parameters of the combination of particles that were injected to the inlet port

\begin{tabular}{lll}
\hline Variables & $\mathrm{RBC}$ and cancerous cell & $\mathrm{Unit}$ \\
\hline Frequency of electrical field & 100 & $\mathrm{kHz}$ \\
Fluid medium conductivity & 55 & $\mathrm{mS} / \mathrm{m}$ \\
Fluid relative permittivity & 80 & \\
Fluid density & 1000 & $\mathrm{~kg} / \mathrm{m}^{\wedge} 3$ \\
Fluid dynamic viscosity & $1.00 \mathrm{E}-03$ & $\mathrm{~Pa}{ }^{*} \mathrm{~s}$ \\
Particle density & 1050 & $\mathrm{~kg} / \mathrm{m}^{\wedge} 3$ \\
\hline
\end{tabular}

As illustrated in Figures 2 and 3, p-DEP force pulls particles toward a higher electric gradient, while n-DEP force repels the particles away from the higher electric gradient. Thus, the various targeted cell with different dielectric properties can be manipulated by adjusting the medium properties or the input voltage condition. The magnitude and direction of the DEP force are determined by the intrinsic dielectric properties of particles and medium. It is expressed in (1) [18]:

$$
\mathrm{F}_{\mathrm{DEP}}=2 \pi \mathrm{r} 3 \varepsilon \mathrm{m} \varepsilon \mathrm{Re}[\mathrm{fCM}] \nabla|\mathrm{Erms}| 2
$$

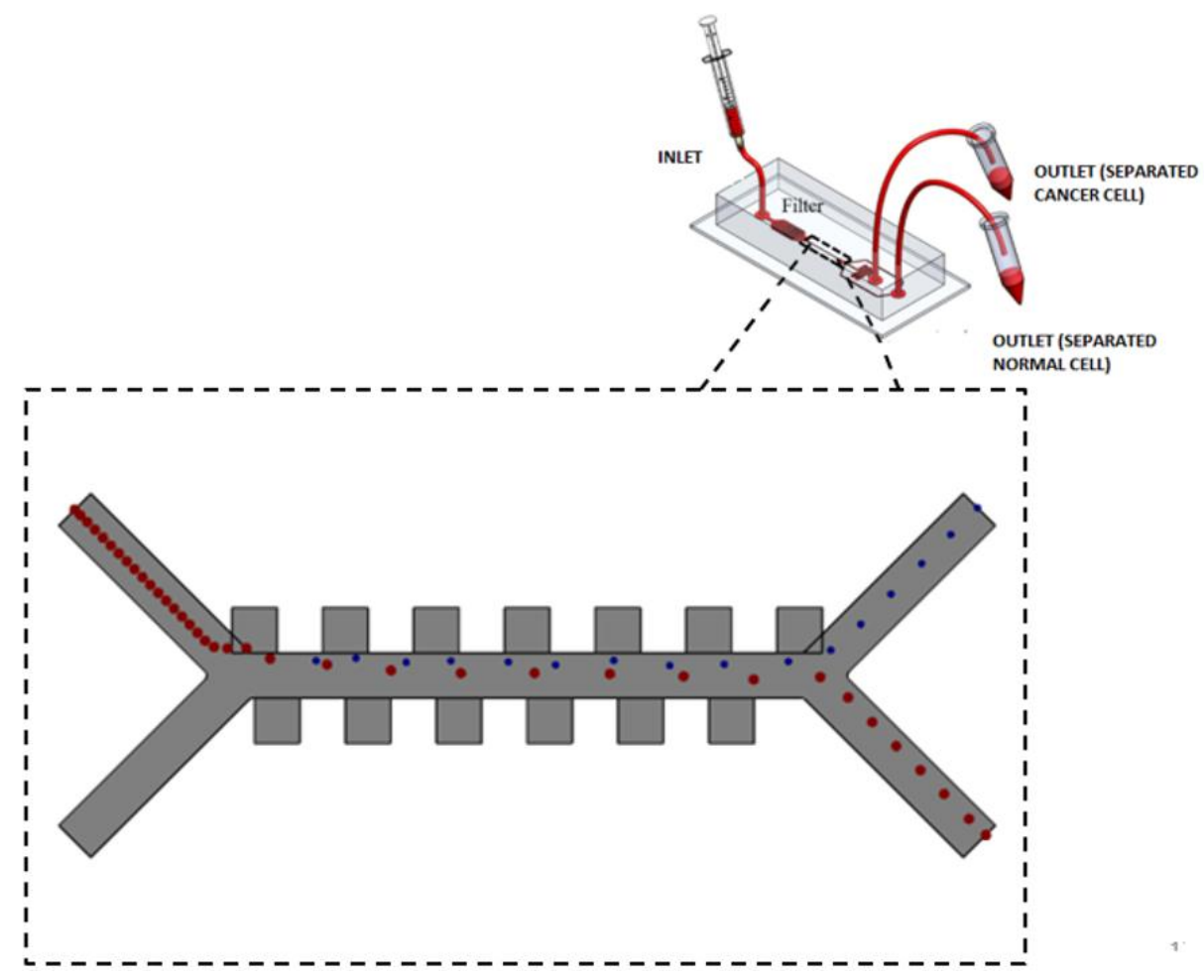

Figure 2. The main channel inside the microfluidic chip [19] 


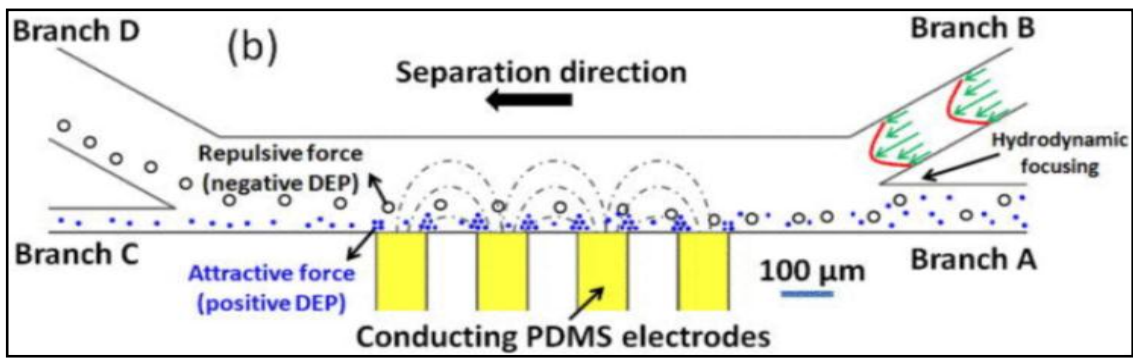

Figure 3. The overview of how dielectrophoresis work [20]

The particle's movement is depending on three forces, which are DEP forces, hydrodynamic force, and gravity [21]. A blood sample will be injected through Branch A while hydrodynamic forces will be supplied at Branch B. Next, the cells will then pass through the main channel and experience sedimentation and levitation due to the gravity and n-DEP Force [22]. When the DEP force is larger than gravity, particles will move diagonally due to hydrodynamic force [23]. After the cells experience the repulsive and attractive force, it will then be separated to Branch $\mathrm{C}$ (an outlet for healthy cell) and Branch D (cancerous cell). The complex permittivity of a living cell can be approximated using (2) [24-26]:

$$
\varepsilon_{p}^{*}=\varepsilon_{\text {mem }}^{*} \frac{\left(\frac{r}{r-d}\right)^{3}+2\left(\frac{\varepsilon^{*}{ }_{i n}-\varepsilon^{*} \text { mem }}{\varepsilon^{*}{ }_{\text {in }}+2 \varepsilon^{*} \text { mem }}\right)}{\left(\frac{r}{r-d}\right)^{3}-\left(\frac{\varepsilon^{*}{ }_{i n}-\varepsilon^{*} m e m}{\varepsilon^{*}{ }_{\text {in }}+2 \varepsilon^{*} \text { mem }}\right)}
$$

where;

$\varepsilon^{*}{ }_{\text {in }}=\varepsilon_{\text {in }}-\mathrm{i} \sigma_{\text {in }} / \mathrm{w}$ : effective complex permittivity of the cell interior

$\varepsilon^{*}{ }_{\text {mem }}=\varepsilon_{\text {mem }}-i \sigma_{\text {mem }} / \mathrm{w}$ : effective complex permittivity of the cell membrane

The membrane of a living cell as in many orders of magnitude less conducting than the cell interior is $\left(\sigma_{\mathrm{mem}} \ll \sigma_{\text {in }}\right)$. Thus, at frequencies well below the Maxwell-Wagner relaxation frequency $(\sim \mathrm{MHz})$, the factor:

$$
\left(\frac{\varepsilon^{*}{ }_{\text {in }}-\varepsilon^{*}{ }_{\text {mem }}}{\varepsilon^{*}{ }_{\text {in }}+2 \varepsilon^{*} \text { mem }}\right) \approx 1
$$

If membrane thickness $(\mathrm{d})$ is very small relative to cell radius $(\mathrm{d}<<\mathrm{r})$, then cell complex permittivity simplifies to (4):

$$
\varepsilon_{\mathrm{p}}^{*}=\frac{\mathrm{r}}{\mathrm{d}} \varepsilon_{\text {mem }}^{*}=\mathrm{r}\left(\mathrm{C}_{\text {spec }}-\mathrm{j} \frac{\mathrm{G}_{\text {spec }}}{2 \pi \mathrm{f}}\right)
$$

where;

$\mathrm{C}_{\mathrm{spec}}=$ Specific membrane capacitance

$\mathrm{G}_{\text {spec }}=$ Specific membrane conductance

\section{RESULTS AND DISCUSSION}

A commercial software package, COMSOL 5.3a was used to produce a designated model to study the flow of the channel. This software has unique capabilities for multiphysics, multiscale and coupled simulations of fluid, thermal, chemical, biological, electrical and mechanical phenomena for real-world applications [27]. The program provided an integrated geometry and grid generation module, a graphical user interface for preparing the model, a computational solver for performing the simulation and an interactive visualization program. These were used to analyze the simulation results.

\subsection{Design of the microfluidic device}

In this study, three designs were proposed. The dimensions and number of input and output are the same. The only difference is the location of the electrodes. All designs were tested to determine the most ideal and effective location of electrodes. To make the visual more clear, the model has been designed in the 2D diagram as shown in Figure 4. In Figure 5 the simulations were tested to investigate the velocity, pressure, and electrical potential of all the proposed designs. 


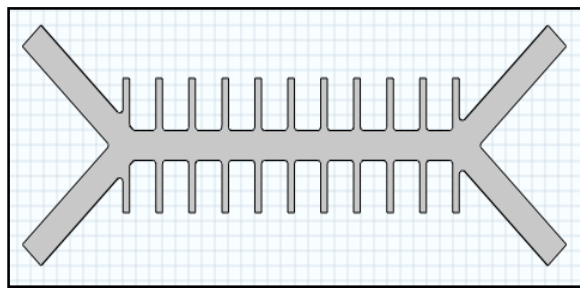

(a)

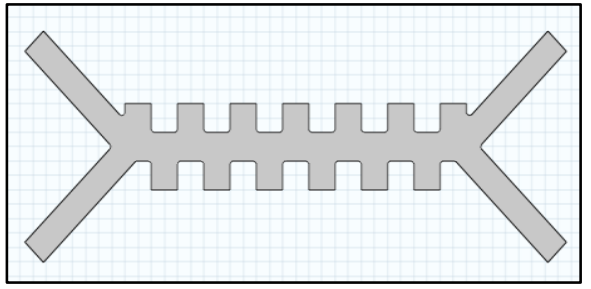

(b)

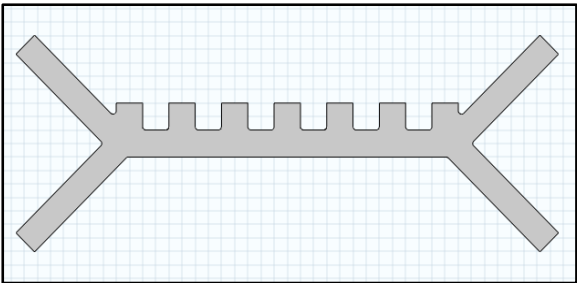

(c)

Figure 4. 2D design of microfluidic device, (a) Parallel electrodes located on the same $\mathrm{x}$-axis coordinate, (b) Parallel electrodes with different coordinates, (c) Electrodes parallel with plate electrode

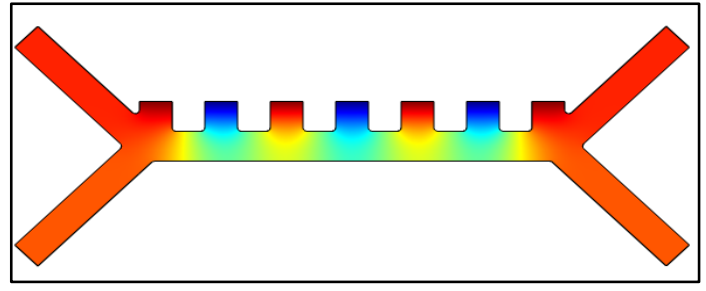

(a)

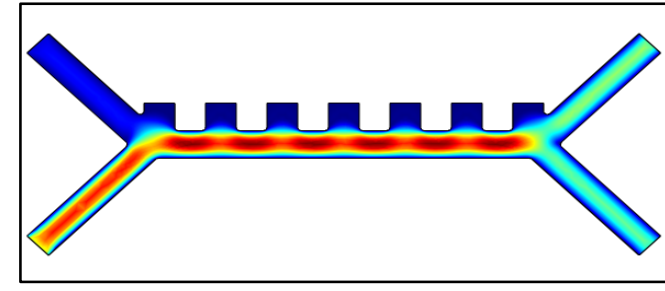

(b)

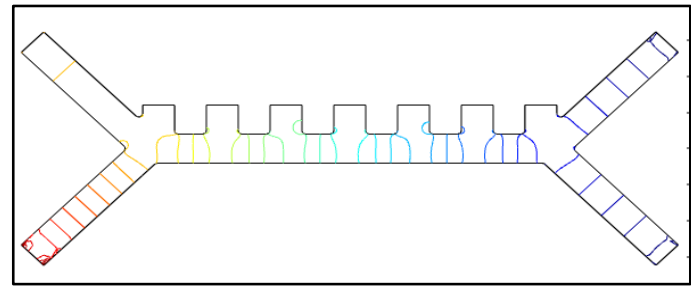

(c)

Figure 5. The simulations in parallel-plate design, (a) The electric potential in the parallel-plate design, (b) The pressure of the parallel- plate design, (c) The velocity magnitude of the parallel- plate design

\subsection{Simulation investigation: Velocity, pressure and electrical potential}

Three studies were tested to obtain the results of cell separation. The first study is to obtain the velocity, pressure and electrical potential. For the first study, all designs were succeeded. The graphic output was the same as the data inserted in the simulation. Next, the manipulated variables in this study are voltages, impedances, and frequencies. The voltages were tested from $5 \mathrm{~V}$ to $25 \mathrm{~V}$. Impedances were in the range of $50 \mathrm{ohms}$ to $300 \mathrm{ohms}$, while for the frequency, the simulation was tested below the range of $50 \mathrm{kHz}$.

\subsection{Simulation investigation: Simulation without dielectrophoretic force}

For the second study, the particle trajectories without DEP force were applied. The blood cells were released at the same time and follow a similar path. In this simulation, the time starts at 0s and end at the third second. At this situation, there was no cell separation because DEP force $=0$. This simulation was succeeded as shown in Figure 6. 


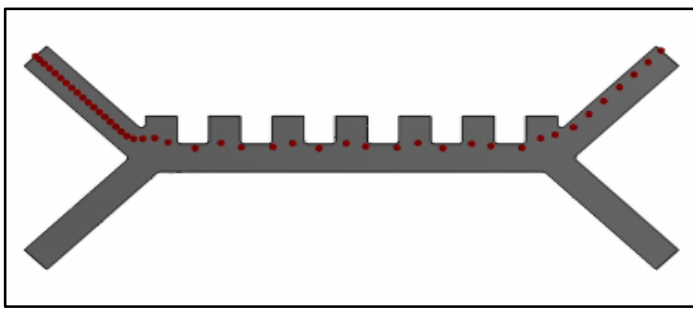

(a)

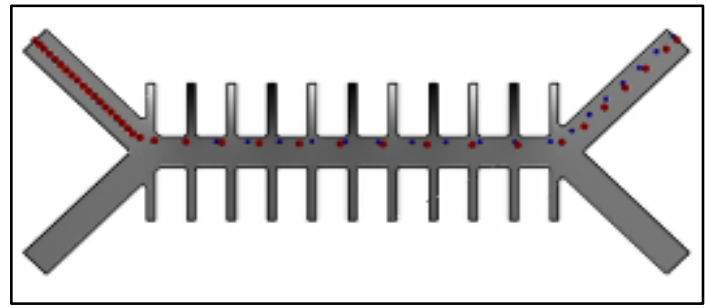

(b)

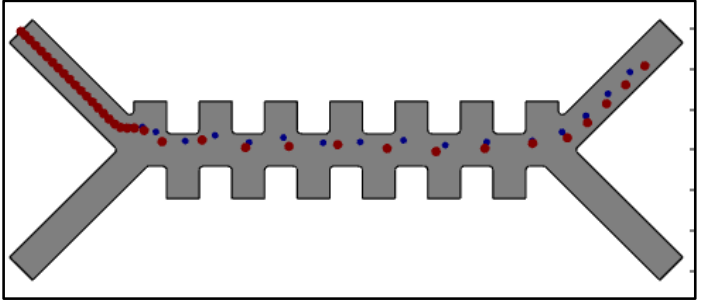

(c)

Figure 6. The design without dielectrophoretic force, (a) Electrodes parallel with plate electrode, (b) Parallel electrodes located on the same x-axis coordinate, (c) Parallel electrodes with different coordinates

After the second study was simulated, results show that all designs took approximately 2 minutes to reach the end of the channel. In this situation, the velocity of the particles is the main role and the most related to the flow rate of particles. The flow rate of all designs was the same because the velocity injected for all the designs were the same. The first inlet (blood cells) has a velocity of $135 \mathrm{um} / \mathrm{s}$ while the second inlet (buffer solution has the velocity of $800 \mathrm{um} / \mathrm{s}$ ).

\subsection{Simulation Investigation: Simulation with dielectrophoretic force}

The objective of the third study is to separate the blood cells into two channels. The particles of the cancerous cells were two times smaller than the blood cells to ease the user to track and differentiate the particles. As shown in Figure 7, the particles that were marked by the red color is the normal cells while the blue color is for the cancerous cells.

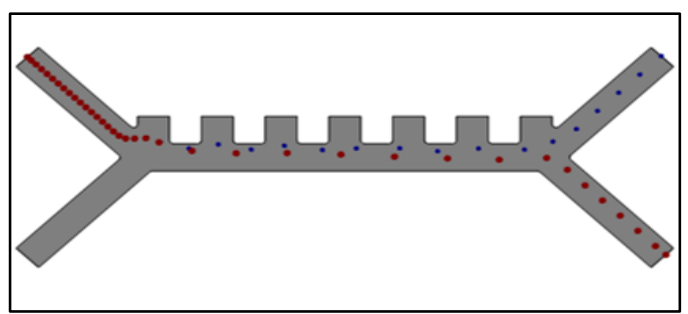

(a)

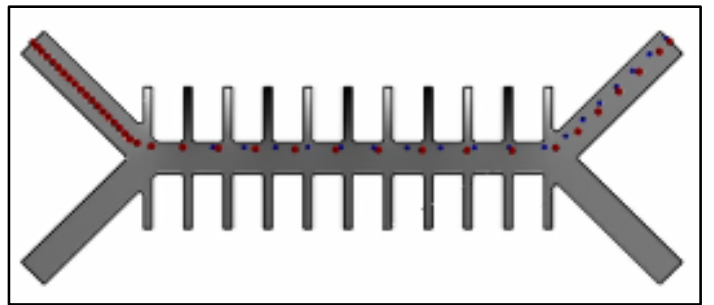

(b)

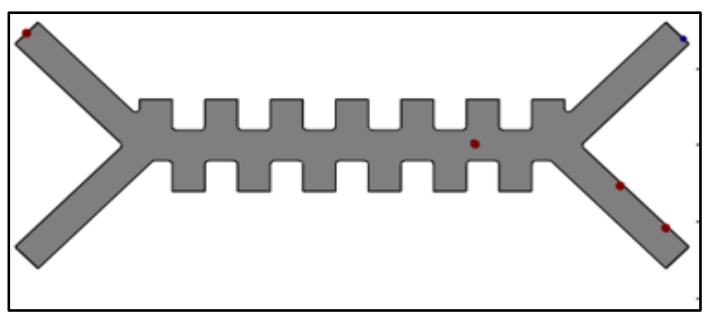

(c)

Figure 7. The design with dielectrophoretic force, (a) Electrodes parallel with Plate Electrode, (b) Parallel electrodes located on the same x-axis coordinate, (c) Parallel electrodes with different coordinates 
The simulation was tested to investigate the movement of particles flow with DEP force and to investigate the time taken and flow rate of particles flow. The same size and shapes of the electrode can cause the charge to be zero, thus there will be no attractive and repulsive force. Positive and negative electrode charges should be designed in different shapes and sizes so that they can produce a different field of charge. In the third study, the flow rate of the particles and the ideal design have been determined. For the first design, the separation time of the particles is around 1 minute and 50 seconds with $5 \mathrm{~V}$ and $10 \mathrm{kHz}$ injected. For the second design, the location of the electrodes to be parallel to each other causing the simulation to fail. The objective to separate the cells cannot be achieved. Same size and shapes of the electrode can cause the charge to be zero, thus there will be no attractive and repulsive force. As for the solution, positive and negative electrode should be designed in different shape and size so that they can produce a different field of charge. Next, for the third design, the separation time of the particles is approximately 1 minute and 4 seconds. Thus, the third design was chosen as the most ideal design of the microfluidic chips

\subsection{Simulation investigation: simulation being tested by variety of voltages, frequencies, and impedance}

The last case to be considered in this study is the variation simulation of the third design. To obtain the maximum value of the electrical properties, the simulation was tested by a variety of properties. The fastest flow rate of this simulation is approximately around 58 seconds with $30 \mathrm{~V}$ and $10 \mathrm{kHz}$. However, the simulation cannot separate the cells when the frequency inserted was above $50 \mathrm{~V}$. This severe temperature increase inside the channel leads to a serious Joule heating effect inside the channel, which can cause the cells to be damaged.

\section{CONCLUSION}

This paper presents the design and the simulation of a microfluidic device that can perform particle separation. This device comprises a PDMS channel with micropillars and a glass substrate with electrodes. The simulations reveal that the particles were successfully captured by the electrodes and sorted within a specific time. The impedance is measured at the frequencies of between 10 to $50 \mathrm{kHz}$. The software COMSOL is adopted to study the flow of the particles in the channel; the probability of the cell capture and the ability of the electrodes to sort out the cells is about $80 \%$. Furthermore, an ideal design of the microfluidic chip, incorporating the cell and dielectric properties have been established.

\section{ACKNOWLEDGEMENTS}

The research was supported by the Ministry of Higher Education of Malaysia and Universiti Teknologi Malaysia (Grant Nos. Q.J130000.2851.00L23 and Q.J130000.21A2.04E82); we thank them for funding this project and for their endless support.

\section{REFERENCES}

[1] H. K. Weir, T. D. Thompson, A. Soman, B. Møller, and S. Leadbetter, "The past, present, and future of cancer incidence in the United States: 1975 through 2020," Cancer, vol. 121, no. 11, pp. 1827-1837, June 2015.

[2] M. Yu, S. Stott, M. Toner, S. Maheswaran, and D. A. Haber, "Circulating tumor cells: Approaches to isolation and characterization," Journal of Cell Biology, vol. 192, no. 3. pp. 373382, 2011.

[3] T. R. Ashworth, "A case of cancer in which cells similar to those in the tumours were seen in the blood after death," The Medical Journal of Australia, vol. 14, pp. 146-147, 1869. In Scientific Research Publishing. [Online]. Available at: https://www.scirp.org/(S(i43dyn45teexjx455qlt3d2q))/reference/ReferencesPapers.aspx?ReferenceID=2005935. [Accessed: 02-Feb-2020].

[4] G. Li and Y. Sun, "Liquid biopsy: Advances, limitations and clinical applications," JSM Biotechnology and Biomedical Engineering, vol. 4, no. 2, pp. 404-408, 2017.

[5] S. Jia, R. Zhang, Z. Li, and J. Li, "Clinical and biological significance of circulating tumor cells, circulating tumor DNA, and exosomes as biomarkers in colorectal cancer," Oncotarget, vol. 8, no. 33, pp. 55632-55645, 2017.

[6] F. Tian, C. Liu, L. Lin, Q. Chen, and J. Sun, "Microfluidic analysis of circulating tumor cells and tumor-derived extracellular vesicles," TrAC Trends in Analytical Chemistry, vol. 117, pp. 128-145, 2019.

[7] Y. Urano, T. Nagano, and M. Sakabe, "Method for diagnosing cancer," United States Patent: US9610366B2, 2017.

[8] A. Kulasinghe, H. Wu, C. Punyadeera, and M. E. Warkiani, "The use of microfluidic technology for cancer applications and liquid biopsy," Micromachines (Basel), vol. 9, no. 8, pp. 397-1-22, 2018.

[9] D. Lee, B. Hwang, and B. Kim, "The potential of a dielectrophoresis activated cell sorter (DACS) as a next generation cell sorter," Micro and Nano Systems Letters, vol. 4, no. 1, p. 2, 2016.

[10] P. R. C. Gascoyne, Xiao-Bo Wang, Ying Huang, and F. F. Becker, "Dielectrophoretic separation of cancer cells from blood," IEEE Transactions on Industry Applications, vol. 33, no. 3, pp. 670-678, 1997. 
[11] C. Loughran and C. R. Keeling, "Seeding of tumour cells following breast biopsy: A literature review," British Journal of Radiology, vol. 84, no. 1006, pp. 869-874, Oct. 2011.

[12] M. He and Y. Zeng, "Microfluidic exosome analysis toward liquid biopsy for cancer," Journal of Laboratory Automation, vol. 21, no. 4, pp. 599-608, Aug. 2016.

[13] M. Ilié and P. Hofman, "Pros: Can tissue biopsy be replaced by liquid biopsy?," Translational Lung Cancer Research, vol. 5, no. 4, pp. 420-423, Aug. 2016.

[14] N. Hao and J. X. J. Zhang, "Microfluidic screening of circulating tumor biomarkers toward liquid biopsy," Separation and Purification Reviews, vol. 47, no. 1, pp. 19-48, 2018.

[15] M. Al Ahmad, Z. Al Natour, F. Mustafa, and T. A. Rizvi, "Electrical characterization of normal and cancer cells," IEEE Access, vol. 6, pp. 25979-25986, 2018.

[16] A. K. Yetisen, M. S. Akram, and C. R. Lowe, "Paper-based microfluidic point-of-care diagnostic devices," Lab on a Chip, vol. 13, no. 12, pp. 2210-2251, 2013.

[17] L. S. Jang and M. H. Wang, "Microfluidic device for cell capture and impedance measurement," Biomedical Microdevices, vol. 9, no. 5, pp. 737-743, 2007.

[18] N. Lewpiriyawong, C. Yang, and Y. C. Lam, "Continuous sorting and separation of microparticles by size using AC dielectrophoresis in a PDMS microfluidic device with 3-D conducting PDMS composite electrodes," Electrophoresis, vol. 31, no. 15, pp. 2622-2631, Jul. 2010.

[19] H. Peng, N. T. Alvarez, C. Kittrell, R. H. Hauge, and H. K. Schmidt, "Dielectrophoresis field flow fractionation of single-walled carbon nanotubes," Journal of the American Chemical Society, vol. 128, no. 26, pp. 8396-8397, 2006.

[20] L. Hajba and A. Guttman, "Circulating tumor-cell detection and capture using microfluidic devices," TrAC Trends in Analytical Chemistry, vol. 59, pp. 9-16, 2014.

[21] J. D. Adams, U. Kim, and H. T. Soh, "Multitarget magnetic activated cell sorter," Proceedings of the National Academy of Sciences of the United States of America., vol. 105, no. 47, pp. 18165-18170, Nov. 2008.

[22] P. Modarres and M. Tabrizian, "Frequency hopping dielectrophoresis as a new approach for microscale particle and cell enrichment," Sensors and Actuators B: Chemical, vol. 286, pp. 493-500, 2019.

[23] P. R. C. Gascoyne, "Dielectrophoretic-field flow fractionation analysis of dielectric, density, and deformability characteristics of cells and particles," Analytical Chemistry, vol. 81, no. 21, pp. 8878-8885, Nov. 2009.

[24] S. Mahabadi, "The investigation of cancer treatments effects on electrophysiological properties of cells by DEP with respect to personalised and stratified medicine," Thesis, The University of Surrey, UK, 2018.

[25] E. A. Punnoose, S. K. Atwal, J. M. Spoerke, H. Savage, A. Pandita, Ru-Fang Y., A. Pirzkall, B. M. Fine, L. C. Amler, D. S. Chen, and M. R. Lackner, "Molecular biomarker analyses using circulating tumor cells," PLoS One, vol. 5, no. 9, pp. e12517, Sept. 2010.

[26] Y. C. Kim, S-J. Park, and J-K. Park, "Biomechanical analysis of cancerous and normal cells based on bulge generation in a microfluidic device," The Analyst, vol. 133, no. 10, pp. 1432-1439, 2008.

[27] M. Zhang, "Permittivity and conductivity imaging in electrical capacitance tomography," Thesis, Department of Electronic \& Electrical Engineering, University of Bath, 2016. 